\title{
A Modular Task Orientated Approach for the Analysis of Large Datasets
}

\author{
Paul Palmer \\ Systems of Systems Research Group, \\ Wolfson School \\ Loughborough University \\ Loughborough, UK \\ p.j.palmer@lboro.ac.uk \\ Russell Lock \\ Computer Science Department \\ Loughborough University \\ Loughborough, UK \\ r.lock@lboro.ac.uk
}

\author{
Michael J de C Henshaw \\ Systems of Systems Research Group, \\ Wolfson School \\ Loughborough University \\ Loughborough, UK \\ m.j.d.henshaw@lboro.ac.uk
}

\begin{abstract}
We introduce a novel conceptual framework using task orientated templates, for the analysis of large data that effectively separates: data curation, analysis, and reporting of large datasets, creating a reproducible analysis. Outputs saved include calculated secondary data, with associated metadata capturing all the transformations that have been applied, to provide an auditable connection to the source data. Data sharing is encouraged by many research funders and academic publishers, but supplying provenance is not mandatory. Enhancing data sharing will benefit many sectors as the pool of trusted data increases and is used with confidence in downstream analysis. While such benefits are likely to initially impact academic research due to active encouragement of data sharing, business intelligence processes will also benefit through increased confidence of source data. Using task orientated templates will allow a more structured approach to data analysis, and facilitate reuse of data through the use of verifiable digital signatures. This template based approach will reduce the programmatic skills required for the analysis large data for a wide range of commercial, academic and social applications on desktop computers.
\end{abstract}

\section{INTRODUCTION}

This paper introduces a novel conceptual framework supporting the analysis of 'Big Data' on desktop computers with limited resources. This framework effectively separates: data curation, analysis and reporting tasks, to build an auditable and repeatable record of such analysis.

The approach is based on the familiar concept of using templates as a foundation for a task, rather than always starting from first principles. Different tasks will require different templates, but each template incorporates essential code and other attributes required to complete the task, but not the data. The aim of this approach is to satisfy the requirement to analyse large data for a wide range of commercial, academic and social applications. Implicit within this requirement is the need to ensure that the analysis is accurate and reproducible, which in this case, is met by the template. This conceptualisation requires task specific templates and hence a modular approach.

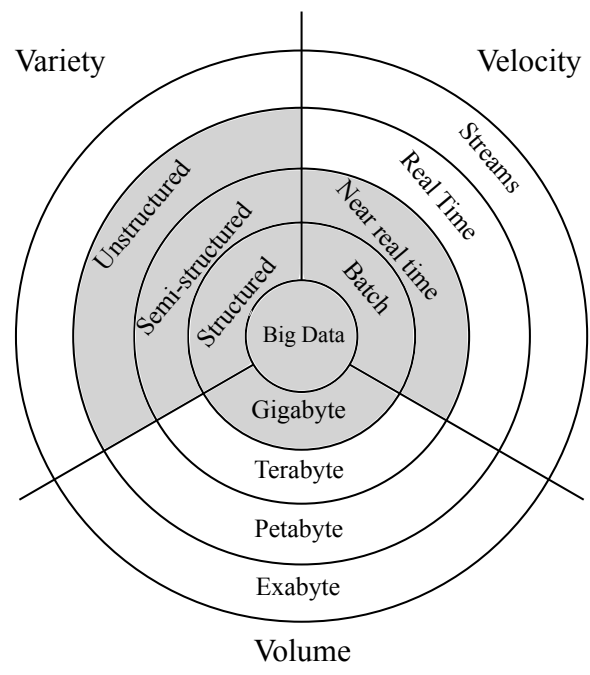

Figure 1: Three V's of Big Data. (After Sagiroglu [2].) Shaded areas are those considered to be within range of desktop computers in this work.

There are so many sources and types of data in the modern world that the term 'Information Age' has become a colloquial term describing this aspect of current human endeavour and the associated growth of data [1]. Before describing the conceptual framework that is the purpose of this paper, it will be helpful to provide context and set boundaries for the scope of this work.

\section{A. Background}

The Information Age is characterised by ever-increasing access to digital data in a phenomenon is known as 'Big Data'. But that data is often in a variety of inconsistent formats created both by automated processes and human effort. 
Sources of data include: Healthcare; Government; Personal Location; Retail; and Manufacturing [3]. Analysing petabytes of such data is seen as 'a hard nut to crack' due to its volume, variety, and velocity [2], [4]-[7] (See Figure 1); the tools for analysis of data on this scale require dedicated technology such as Apache Hadoop, Chip Multi-Processors (CMP), and Fast Array Wimpy Nodes (FAWN) [8], [9]. However, data comprising many millions of rows may occupy only tens of gigabytes in storage space, and while beyond the current capability of familiar tools such as spreadsheets [10], the data may be analysed on desktop computers with alternative programmatic tools [11].

Big Data are not usually collected as part of a designed experimental methodology, nor are parameters selected for the statistically validated testing of a hypothesis. 'Collect data first, ask questions later' is a more likely analytical approach [12], leading to the use of more exploratory knowledge discovery techniques. This in turn has lead to an increased interest in the application of non-parametric methods for data visualisation, such as probability density estimation [13]. Understanding how such methods scale with size of data becomes a key question when determining what may be achieved with the ever-increasing power of the desktop computer.

\section{B. Desktop analysis}

This work limits itself to challenges of analysing data within the shaded area of Figure 1. This scope should be interpreted as a minimum current capability of desktop computers, and is a limit that is continually moving outward as technology advances. So fast is this progress, we do not try relating it to any hardware or software specification, as that seems more likely to over constrain the application of concepts presented in this work. Drivers for adopting 'in-house' analysis are not likely to be technical. Indeed, issues such as cost, confidentiality, or commercial sensitivity may make in-house analysis desirable, while to academics, complete control of all stages in the analytical process facilitates reproducible research.

Figure 2 provides a coherent framework through which the concepts discussed in this paper are contextualised. It is a conceptual process that provides only a high-level view and each process stage can be regarded as isolated, so that they may be considered to be replaceable modules that supply or transform data, or output the results. This conceptual process is developed into a task oriented template approach in section IV . Note that an output may take many forms other than a summary, visualisation or even transformed data that may feed into further analytical steps making the overall process recursive.

Elaborating each process step in Figure 2:

- Data. Data may comprise one or more datasets and (ideally) includes metadata describing structure of the dataset.

- Extract. Extracting the subset of data required for the analysis.
- Load. The process of reading the data into an electronic format ready for manipulation is transformative, so should be included as a process stage. Properties such as character set may be deliberately or inadvertently changed and data-types imposed.

- Transform. Transform the data into the required system format. By this we mean a form that can be manipulated by the analytic software of choice, which may be different to the form in which the data is stored.

- Clean Resolve problematic data. Strategies used are task dependent and will address issues in the data, such as incomplete, out of range, duplicate and other inconsistencies.

- Analyse. The analytical process is predicated upon the outputs of preceding phases and depends upon the analytical intent of the user.

- Report. The final output, which will be in a human readable format. Note that this may also include supporting data files.

In this work we build upon this conceptual process to explore an approach that reduces the coupling between user skill and computational efficiency when working with large datasets. We show how data from very different sources can map onto an abstract data classification taxonomy, such that the analytical approaches required to extract information are identical, or at least very similar. These observations are built upon to define modular analytical templates that are applicable across multiple datasets that share a similar structure. We start by discussing attributes of Big Data in Section II and discuss current practice in metadata, loading, and transformation. Transformation is here taken to include cleaning. In Section III we discuss current analytical practice and its limitations. In Section IV, we introduce our approach and describe its advantages, and finally in Section $\mathrm{V}$ we discuss key issues for future work.

\section{BIG DATA}

\section{A. Defining Big Data}

There is no universally agreed upon definition of Big Data. One group of researchers, Fosso et al, cite ten plausible definitions [14]. Also, many authors implicitly choose to examine Big Data from a database perspective. For example, Elgendy et al [15] state without explanation, that dataset size is the most important characteristic of Big Data. Closely related to this topic are the widely cited, often without attribution: 'n V's of Big Data': Volume, Variety, Velocity, Value, Veracity etc. etc. We suggest that the unifying thread that makes sense of all these different definitions is to consider a task orientated perspective. That is to say, the challenges of big data analysis depend upon the analytical task. This also implies that the same datasets may be classified differently if the analytical intention changes. This acknowledgement of multiple perspectives helps to inform the choice of definition for Big Data. We favour the following definition: 


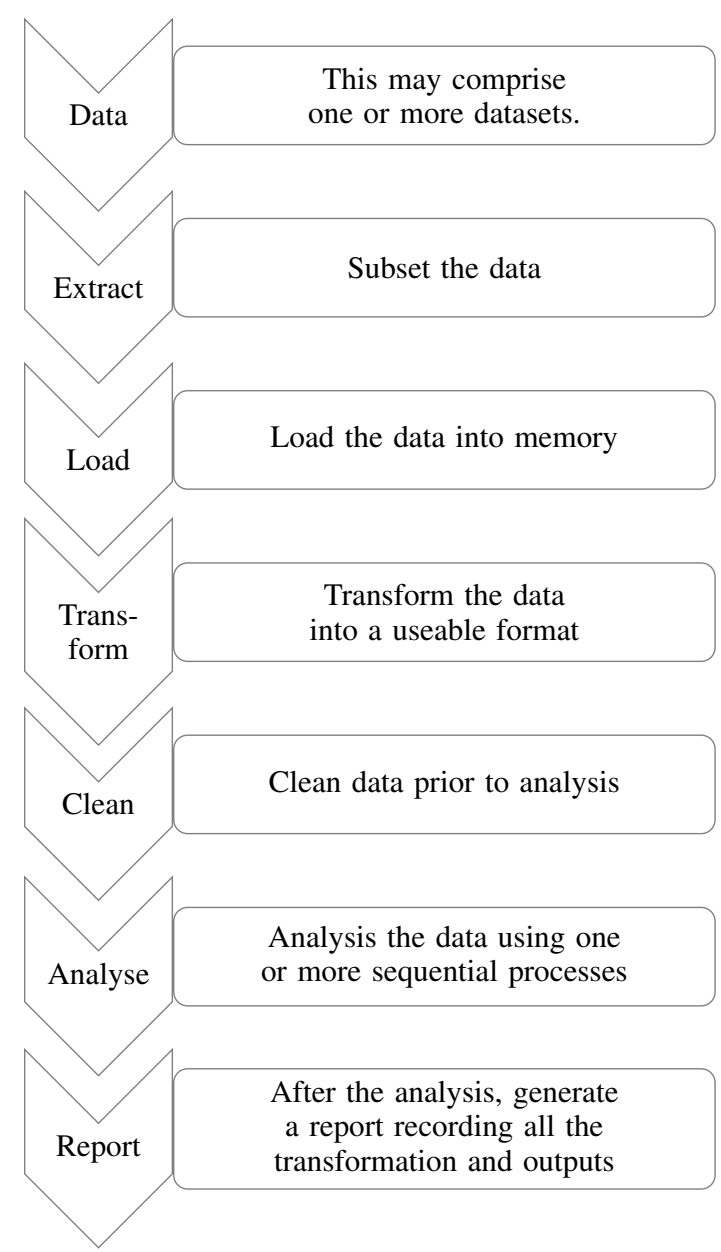

Figure 2: Conceptual analytical process. Extract, Load, Transform, Clean, Analyse, Report

Big Data: data that cannot be handled and processed in a straightforward ${ }^{1}$ manner [16].

A task orientated definition of this form seems appropriate given the wide range of challenges associated with Big Data. It also carries a sense that the challenge of Big Data is analytic in nature and not just a matter of data size. The other definitions may be considered as describing useful characteristics appropriate to the particular tasks under consideration.

\section{B. Metadata}

Metadata is always required to guide the subsequent loading and cleaning activities, otherwise suitable actions will have to be inferred from the data contents with the accompanying risk of errors. While there are no universally accepted standards for metadata, various de facto standards are in use by national agencies and organisations that promote the exchange of information. The creation of machine-readable metadata standards would be an important step in the construction of automated loading of data and its interpretation. An abstract classification of data type proposed by Shneiderman [17] in

\footnotetext{
${ }^{1}$ Straightforward: Easily analysed with common desktop computer tools.
}

1996 remains relevant today as a task by data-type taxonomy, where it forms a useful component of metadata:

1) 1-dimensional: linear data types include textual documents, program source code, and alphabetical lists of names which are all organized in a sequential manner.

2) 2-dimensional: planar or map data include geographic maps, floor-plans, or newspaper layouts. Each item in the collection covers some part of the total area and may be rectangular or not.

3) 3-dimensional: real-world objects such as molecules, the human body, and buildings have items with volume and some potentially complex relationship with other items.

4) Temporal: time lines are widely used and vital enough for medical records, project management, historical presentations to create a data type that is separate from 1-dimensional data. The distinction in temporal data is that items have a start and finish time and that items may overlap. Frequent tasks include finding all events before, after, or during some time period or moment, plus the basic tasks.

5) Multi-dimensional: most relational and statistical databases are conveniently manipulated as multi- dimensional data in which items with $\mathrm{n}$ attributes become points in a $\mathrm{n}$-dimensional space.

6) Tree: hierarchies or tree structures are collections of items with each item having a link to one parent item (except the root). Items and the links between parent and child can have multiple attributes.

7) Network: sometimes relationships among items cannot be conveniently captured with a tree structure and it is useful to have items linked to an arbitrary number of other items. While many special cases of networks exist (acyclic, lattices, rooted vs. un-rooted, directed vs. undirected) for simplicity, they are grouped here as a high level family. We recognise that further subdivision may be useful in the analytic process.

\section{Loading data}

The initial loading of data is often wrapped together as an ETL (Extract Transform Load) process as in Figure 3. Big Data brings additional challenges to this process especially due to the volume and variety of the data [18], [19]. As stated in Section I, this work focuses on the challenges of analysing Big Data on desktop computers, so that an assumption is made that an extraction process has already reduced the volume of data to a manageable size. Data may also be stored in many physical formats ranging from paper copies to digital. Character encoding may also be an issue resulting in 'junk' characters appearing within the data. Proprietary formats such as Microsoft Excel .xlsx, even though very popular, may be unreliable due to changes in the internal format [10].

\section{Cleaning data}

A dataset may be 'dirty data' and require cleaning, in that it may contain one or more inconsistencies that present obstacles 
to its use. Cleaning applies transformations to data that should be reproducible like any other analytic process. Metadata may inform on the expected content and guide the process of cleaning by helping to identify non conforming data. Many authors note that data cleaning may be a very time consuming process due to complexity. For example, over thirty types of dirty data are proposed in the taxonomy by Kim et al. [21]. A condensed list of this terminology is provided here to illustrate common issues that must be accommodated prior to analysis:

- Incorrect (Wrong, False, misleading).

- Incomplete (Missing data).

- Untidy (Inconsistent terminology or units).

- Ragged (Irregular matrix rows or depth).

- Inconsistent format. (e.g. a single column may contain a mixture of text and numeric values).

- Amalgamation (of several related datasets that use different terminology).

- Systematic errors.

- Inconsistent content. (e.g. empty columns of data may be automatically removed or re-ordered for reasons that are opaque to the end user).

Multiple authors agree that data cleaning is an under researched topic, and concisely describing the issues is a useful first step in understanding how the data may be cleaned prior to analysis. While this taxonomy Kim et al [21] does not represent a definitive standard, it does cover a broad spectrum of data issues, so provides useful vocabulary with which to describe the issues that required corrective transformations to be applied to data [22]-[25].

As suggested above, the data cleaning process is predicated upon the assumption that a transformation (colloquially called 'rinsing') may be applied to a dirty dataset to derive a clean data-set. Common techniques used include:

- Ignore incorrect or incomplete data.

- Interpolate incomplete data with estimated values.

- Rescale mixed units and recode synonyms.

- Expand observations with additional depth (derive additional columns).

- Recode inconsistent formats. E.g. dates, geocoding.

- Append datasets from multiple sources (additional rows).

- Join data (additional columns and rows).

The selected cleaning strategy will depend on both data and analytical intent. For example, simply ignoring incomplete data may be appropriate if it can be shown that no unintended bias is introduced. If an important subset of data has observa-

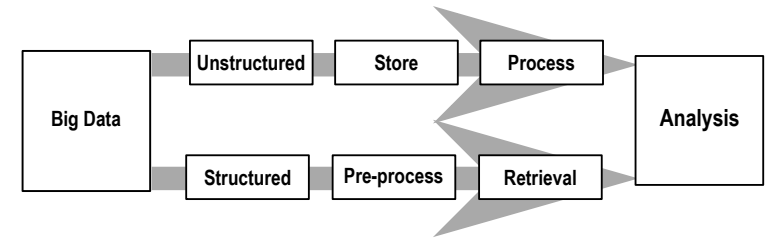

Figure 3: Typical Big Data ETL process (After Hashem [20] ) . tions with a random missing value, then interpolation of the values may be a more cleaning effective technique. Statistical, rule based and learning systems which may help automate the process when applied to the Big Data context, have been proposed [26]-[28], and a number of data cleaning tools also exist [23], [25], [26], [28]-[31] which may be applied as an external data cleaning process. Such tools are clearly useful additions to the analytical toolkit and it is easy see their utility as a precursor to analysis with popular desktop software. However, recording the data transformations these external tools introduce into a systematic and reproducible analytical process is challenging. We review the most popular tools used in the analysis of Big Data in the following section. This helps to set an end user view-point to the analytical process and provides the context into which our template based approach can be used to improve current practice.

\section{Current Analytical Practice}

The four most popular tools used by data scientists for Big Data analysis in 2016 were reported in one survey as [32]:

1) $\mathrm{R} 48 \%$.

2) Python $45 \%$

3) SQL $35 \%$.

4) Excel $32 \%$.

These summary results indicate that multiple tools are used by data science practitioners and that two programming languages $\mathrm{R}$ and Python are very popular, but not universal. Both $\mathrm{R}$ and Python are well-supported open-source languages that run on all major operating systems. While Python is an excellent general purpose language, $\mathrm{R}$ is optimised for data manipulation making it a good choice for Big Data analysis [33]. However, $\mathrm{R}$, like all such languages, relies upon the technical expertise of the programmer to write efficient code that will execute quickly. Poor code choices can increase the execution of an analysis script by orders of magnitude when compared to efficient coding technique [34].

In the following subsections we consider some analytical approaches that support reproducible analyse using $\mathrm{R}$ and Python. Those considered here are not an exhaustive list, but instead represent techniques that are accessible with supporting academic literature [35], [36].

\section{A. Report generation}

Questions around the reproducibility of research have raised interest in techniques that combine report narrative, analytic code, and data into a single transparent process [37]. Stodden [35] focusses on the importance of good statistical practice when analysing data and cautions that all calculations should be presented with a route back to original data, so results may be verified. In the UK, the main funding bodies now require data created during publicly funded research to be made available for future analysis e.g. [38]-[40]. The UK Data service $^{2}$ is one of a growing number of research council recognised repositories that may be used for the sharing of

\footnotetext{
${ }^{2}$ https://discover.ukdataservice.ac.uk/
} 
data. There is also a trend towards government data to be shared on an open licence, but current research shows that the goals of availability and quality of such data are not always met [41]. Sharing the code used to analyse data is less common but is done within some scientific communities. ${ }^{3}$

No research has been found that review the security and integrity of code and data in the context of open access academic publication. For example, many commercial and open source executable code and scripts are digitally signed to verify provenance [42] [43]. We suggest that, In principle, a similar system could be extended to ensure that code and data shared for research has not been mischievously altered [44]. With these cautionary notes in mind, there are a number of tools that may be useful for supporting reproducible research with Big Data. These are discussed in the following subsections.

1) The Jupyter Notebook: The Jupyter Notebook is an open-source web application for sharing documents with live code, data and narrative. Although the application interface is browser based, it can also be used on a desktop computer with a web server running on localhost [45]. Initiating local access to a Jupyter Notebook and server requires a command line interface. Such access is frequently restricted on company maintained computers as a security precaution. However, Jupyter Notebooks are already an established way of sharing code and data within the astrophysics community. An example of this is the first confirmed detection of gravitational waves from a black hole merger [46]. Supporting Jupyter Notebooks containing the code and data are freely available [47]. Using Jupyter Notebooks requires a high level of programming skill in one of the supported languages, which limit accessibility to those with such skills. However, once written, no special skills are required to access the Notebook or use any interactive sections within it. It is possible that the template approach that we propose may be compatible with the Jupyter Notebook.

2) Dynamic documents using ${ }^{2} T_{E} X$ : ${ }_{\mathrm{LT}} \mathrm{EX}$ is a document software frequently used in the preparation of technical and scientific documentation, although it is suitable for many typesetting tasks. ${ }^{4}$ It differs from popular WYSIWYG word processors in that the text is marked up with tags which are used by a separate template when the document is compiled into its output format. This allows document authors to concentrate on content, and to use templates created by typesetting experts, effectively decoupling the two parts of the document creation process. Latex templates are available for many technical journals [48] and other formal documents. ${ }^{5}$

An extension of this system allows dynamic content to be created by embedding $\mathrm{R}$ code inside a $\mathrm{HT}_{\mathrm{E}} \mathrm{X}$ document and executing the code at compile time. Two LTEX packages 'Sweave \& knitr', are used to integrate $\mathrm{R}$ code into the document flow. Code is evaluated at compile time, effectively separating narrative, code and data into separate documents. In the following example, Listing $1, \mathrm{R}$ code is executed

\footnotetext{
${ }^{3}$ The Astrophysics Source Code Library http://www.ascl.net/ is a notable exception.

${ }^{4}$ See: https://www.latex-project.org/

${ }^{5}$ See https://www.latextemplates.com/
}

at document compilation time. Note the special tags at the beginning and end of the listing that are used to indicate the beginning and end of executable code. The switches, echo, cache and eval control the final appearance of the results.

Appropriate code can be used to insert: graphics, charts, tables, and other objects into the final document. While this approach is versatile, it requires good $\mathrm{R}$ programming skills to make it work. Edits need to be applied to the source file which must then be recompiled to build a new output. This workflow is acceptable for small documents, but will quickly become cumbersome as the page count and output complexity increases.

$<<$ echo=TRUE, c a che=TRUE, eval=TRUE $>>=$

\# Create a sequence of numbers

$X=2: 10$

\# Display basic statistical measures

summary $(\mathrm{X})$

@

Listing 1: $\mathrm{R}$ code embeddded in a $\mathrm{LT}_{\mathrm{E}} \mathrm{X}$ document for evaluation at compilation.

An alternative approach is to embed controls in the form of comments within an $\mathrm{R}$ script that direct the format of an output document. $\mathrm{R}$ markdown is a package that can be used in conjunction with any $\mathrm{R}$ compiler and enables document structure, narrative, and $\mathrm{R}$ code to be inserted within an $\mathrm{R}$ script. One, quite natural way of working with $\mathrm{R}$, is to explore data by executing short stanzas of $\mathrm{R}$ code, building the analysis as a series of steps. Markdown effectively enables the comments to become part of the output when the file is subsequently processed to make the final document. Any substantive edits to the output need to be made to the $\mathrm{R}$ source file before recompiling to build a new output file. This is a versatile approach which offers a high degree of integration between program and narrative. However, as with the previous method, it requires good $\mathrm{R}$ programming skills to make it work. See Listing 2.

The preceding methods of dynamically producing output documents require good programming skills to make them work. In each case the user is required to generate markup code which works in conjunction with a compiler and optional template files to produce the final output in the desired format. None of these methods use the equivalent of an expert designed template to guide the analytical part of the process, which is entirely left to user skill. All three methods can be used to generate $\mathrm{AT}_{\mathrm{E} X} \mathrm{Xoutput}$. This is emphasised because LTEXdocuments can be transformed into many other formats using Pandoc (which itself supports templates to guide output format) [49], [50].
\# A Sample listing
This is a simple example of an embedded code chunk.
“ ‘ $\{\mathrm{r}$, echo=FALSE $\}$
\# Create a sequence of numbers 


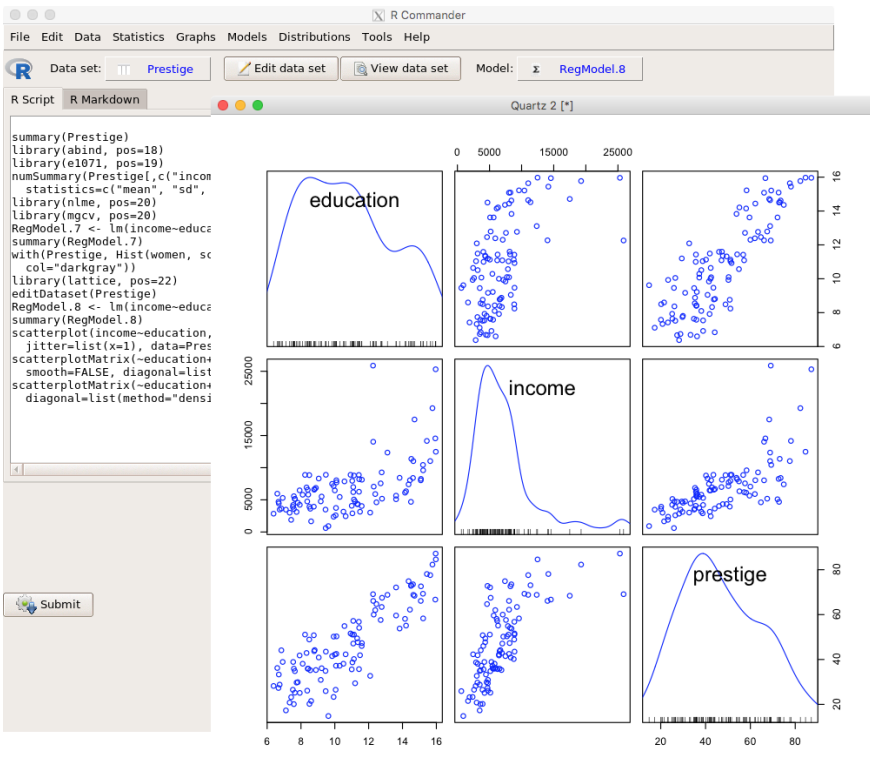

Figure 4: The R Commander GUI provides a menu-based interface for $\mathrm{R}$ functions. The representative output shown here has been generated by selecting menu option. The equivalent $\mathrm{R}$ code is shown in the background.

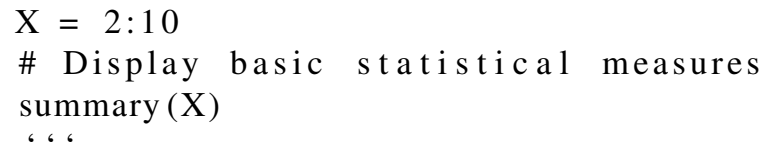

The code will be evaluated at compile time. Listing 2: Markdown document with embeded R code chunks executed at compilation.

\section{B. $R$ Commander}

$\mathrm{R}$ is not shipped with a GUI interface, although there is support for producing dialog boxes within $\mathrm{R}$ using $\mathrm{Tcl} / \mathrm{Tk}$ [51], but there is no overall framework in which to develop a GUI based on this package. R Commander is notable amongst the available third party GUis in that it is Open Source and may be easily installed from within the $\mathrm{R}$ console [52], [53]. It provides a menu style framework that may be extended by writing standard $\mathrm{R}$ packages that conform to the $\mathrm{R}$ Commander extensions [54], [55]. It will run on any system with a correctly installed $\mathrm{R}$ application and will also run alongside many other interfaces including the popular $\mathrm{R}$ Studio IDE. The $\mathrm{R}$ Commander package structure offers access to menu selectable statistical tests and plots via a point and click interface [56], as illustrated in Figure 4. The sample output shown illustrates the generation of matrix scatter diagrams against three variables, accompanied by a frequency density plot. Such diagrams are frequently used as a first step in understanding data. A further sophistication is the control of access to menu options through the context related 'data models', to prevent inappropriate selection. Although originally conceived as an educational software, its flexibility and compatibility offer potential beyond this limited role. In principle, it may be used as a GUI in conjunction with any of the preceding interfaces given the creation of suitable packages to guide its use. Indeed, some users may find that $\mathrm{R}$ Commander is a sufficient tool for their analytical requirements. Support for R markdown, discussed in SectionIII-A2 above, is built-in to R Commander enabling the output of a report capturing the calculation steps

In section IV we propose a new method that also separates the data, analysis, and reporting into separate subsystems of the overall process to provide this type of flexibility.

\section{A TASK ORIENTATED APPROACH}

\section{A. Overview}

Figure 5 presents a modular concept for analysing data and generating a skeleton report ready for the inclusion of a descriptive narrative. The major modules are described in the sub-sections that follow.

The generic task is taken to be an analysis of one or more datasets and the construction of a report containing charts and tables as an output. The analysis code and the data sources are included as appendices. In reality the appendices may contain pointers to permanent public data repositories, rather than physically include the actual data and code within the report. Academic journals are tending towards this approach [40], [57]-[59].

The report is an editable $\mathrm{LT}_{\mathrm{E}} \mathrm{X}$ document linked to programmatically generated content. It is intended that this report may be updated when the template or data is modified without destroying descriptive edits made by the user. This approach separates the data, coding and report elements into separate files. Importantly, it does not require mixing report related comments in the code, or code blocks within the report. This recognises that different skill sets may reside in different team members so is more suited to collaborative working, or reuse in other situations.

\section{B. Data}

The data block in Figure 5 captures the provision, curation and description of data and builds upon the concept presented in Figure 2. Even with a cursory examination of real data, it becomes clear that many, or even most, data require cleaning before use, highlighting the importance of all initial data transformation steps. Transformations acting on data are included within the adjacent Modular analysis template. Associating successful transformations with metadata will facilitate future analytic re-use by other workers. To clarify: creating a secondary dataset where all the transformations that have been applied to the source data, have been captured as metadata, adds provenance to the secondary data and provides an auditable connection to the source data. We suggest that provenance is required to allow the secondary data to be used with confidence in downstream analysis.

\section{Metadata}

As briefly discussed in Section II-B, metadata provides additional information about the data, and is often needed to 
Source

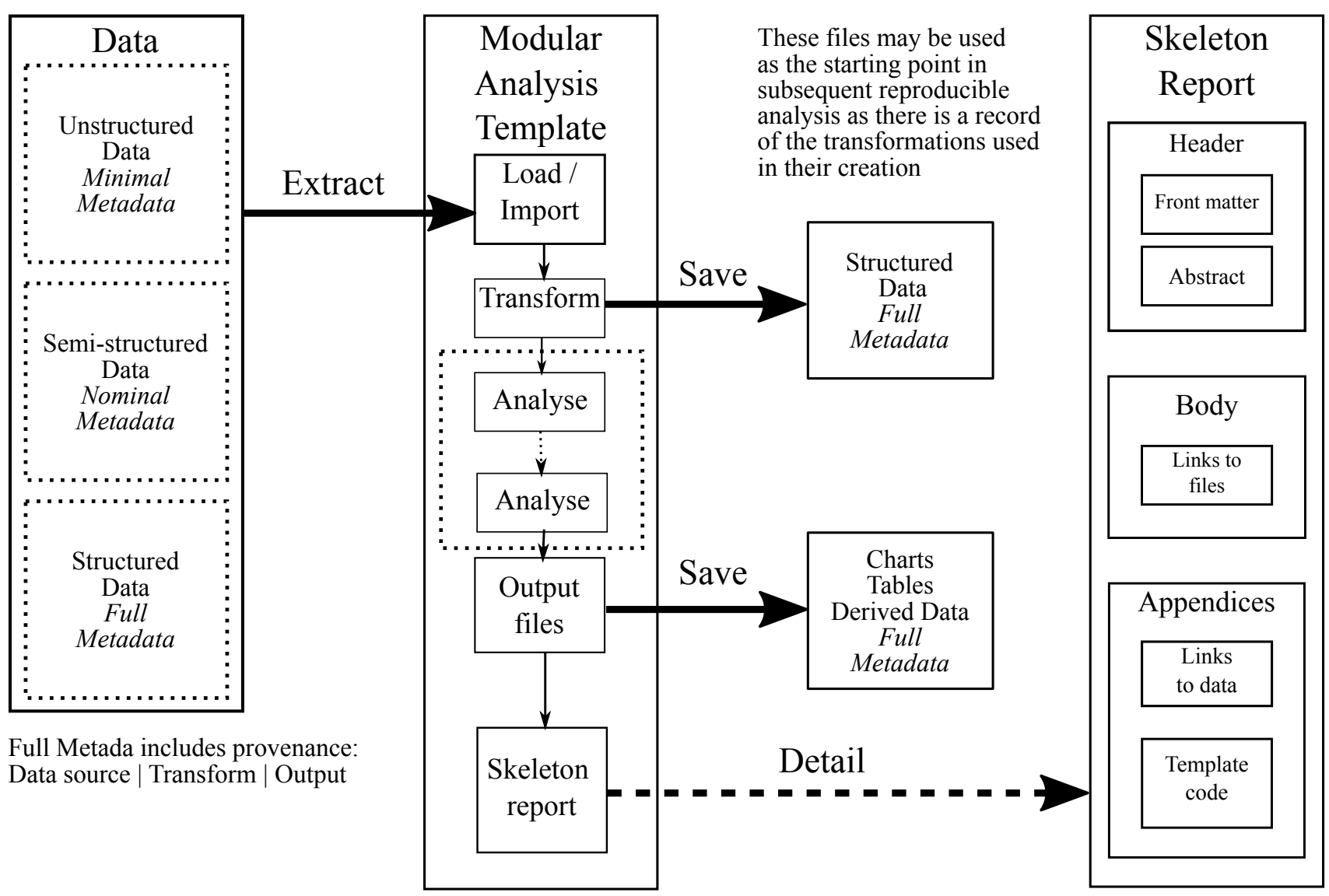

\section{Output}

These files may be used the starting point in analysis as there is a record of the transformations used their creation

Figure 5: Modular analysis template concept.

disambiguate different interpretations of fields within the data. A desirable requirement, (essential for some applications), is that the metadata be machine-readable. We probably do not need to look any further for an explanation as to why incomplete metadata are so commonly encountered. We discuss here how capturing metadata may be built into the analytic process and how it can facilitate data sharing and reproducibility.

Within Figure 5, we illustrate that source data may range from unstructured with minimal metadata, to structured with full metadata. In each case the data is loaded, transformed as required, and made available to the analytic process. At this point we have a software useable version of the source data, knowledge of where it came from, and the transformations that have been applied. It is a relatively small additional step to save this version of the data with metadata capturing implicit and explicit knowledge. We introduce here the need for this to include data provenance, which is taken to include the unique identification of the: data source, all applied transformations, and the output. Various techniques have been proposed for capturing data provenance to support sharing [60]-[62], along with schemes to describe transformations in a platform inde- 
is cautious, so starts by taking the source data and Alice's script to check the outputs are indeed the same. Sure enough, the resulting hash is identical, so Chris is now happy to accept this data from Alice and use the hash values as references.

This scenario illustrates what may be achieved with a pragmatic approach to verifying provenance. We argue that recording the sequence of verifiable identifiers for data and code is sufficient to provide provenance without the need to use complex schemes to capture the details of data and applied transformations.

\section{Modular analysis templates}

The proposed modular analysis template in Figure 5, captures all the programmatic elements into a single package comprising one or more files. The purpose of this is to condense the need for programmable skills into a single template that may be reused on similar or different data. Essential features of the template are:

- The template is designed as a reusable package.

- It contains all the code that extracts, loads, transforms, analyses, and outputs files.

- The template and its contents are uniquely identifiable with a verifiable hash.

- Individual components within the template are identifiable with a verifiable hash.

- Outputs are identifiable with a verifiable hash.

- Hash values are managed to maintain provenance.

- Metadata is captured as a function of the template.

Thus, the template is a skilfully crafted package, and comparisons may be drawn to the templates and 'Wizards' that are in common usage with much software. The skill required to craft a template is much more demanding than the skill needed to reuse one.

We now consider each of the blocks within the modular analysis template in turn.

\section{E. Analysing data}

In section III we discussed current analytical practice. We now discuss the advantage of using a template as a container for the analytic code. As in the earlier section, our discussion here is abstract, so details of the actual analysis are not required. The code within the template file contains all that is needed to perform a particular analysis with the data that is made available. Depending upon the sophistication of the template, tests may be included to verify the validity of analytical approach. However, it is not intended that templates are an alternative for good mathematical and statistical practice. It is intended that templates are created cover the needs of specific tasks, and this is reflected in the title of this paper. To elaborate, with free-form development of code reuse with new data can be time-consuming, leading to code authors creating custom packages, or even copying and pasting code from personal libraries. We can offer no academic papers describing such practice, but evidence may be found by searching online forum postings by experienced coders. ${ }^{7}$

\section{F. Output files}

As described in the introduction to this section and Figure 5, the template code is used to output files including charts, tables and derived data, each identified with a unique verifiable hash. Creating derived data which has provenance connecting it to source data is potentially a valuable resource for promoting reuse requirements introduced in Section III-A, as it now has full metadata and is well-structured for downstream analysis.

\section{G. Skeleton report}

Within the skeleton report all the dynamic content is inserted as links, so edits to the narrative will be preserved when the dynamic content is updated. Thus, we have a powerful mechanism for separating data, analysis and narrative into separate auditable components. As this output document is intended for editing, it is not uniquely identified with a hash. However, the resources it links to are identified in this way, including structured data files. Thus, the links to data and template code are here seen as included in the appendices along with their identifying hash values. We note here that we have not yet found any examples of academic data repositories signing or hashing data as proof of provenance, accompanying the permanent DOI links.

In truth, the actual file format of skeleton report matters much less than compatibility to a wide choice of editor software. Thus, open formats are highly desirable and while LTEXwould be the author's first choice, the extra step of producing outputs in multiple formats is technically trivial. The Open Source tool Pandoc [49], can transform the output into many other formats, including: MS-Word, OpenOffice, epub (electronic book), and html. The skeleton report is therefore to be seen as a foundation to support the inclusion of good quality narrative, rather than the final output.

We have not attempted to incorporate a versioning system such as GIT or Subversion into this narrative, but we note that support for the versioning of large files is improving reducing the barriers for their use. A practical implementation of version contr

\section{Discussion}

There is justifiable debate on the definition of Big Data, but the simple one used in this work: 'data that cannot be handled and processed in a straightforward manner', fits well with the task oriented approach to the classification of data proposed by other authors. Such an approach has an advantage in that it recognises the effect of the analytical goal on other stages of the analytical process. While tools exist to help the expert user to mix $\mathrm{R}$ code and comments to make complex reports currently there is no effective method to capture that expertise into a template that can be reused without programmatic skills on related sets of data.

\footnotetext{
${ }^{7}$ For example: https://hilaryparker.com/2014/04/29/writing-an-r-packagefrom-scratch/
} 
Figure 5 schematically captures our proposed approach. We have left open details of the implementation, but suggest the creation of a new $\mathrm{R}$ package to enable complex output to be built conditionally on the dataset used. By condensing all the programming into a single document, we enable the reuse of these templates by others less skilled in programming. Choosing $\mathrm{LT}_{\mathrm{E}} \mathrm{X}$ will enable the output to be formatted using the wellestablished tools and style templates already in popular use. It also facilitates the use of format conversion tools to transform the output into many other formats. Note too, the intention to capture a description of all the transformations applied as metadata and the facility to save secondary data. As stated in Section IV-B, we suggest that providing provenance for data will support reuse with confidence in subsequent analysis. Realising these aspirations is not without difficulty, and we recognise four substantive challenges in moving forward and developing this modular task orientated approach for analysing large datasets:

- Machine readable metadata describing data and transformations applied for analysis.

- Data cleaning strategies guided by metadata, algorithms and other automated methods.

- Analytical templates that may be developed by experienced programmers and used by others without programming skills.

- An editable skeleton output report with links to dynamically created content conditionally driven by the datasets used.

We also recognise that we have built upon the substantive work of others, and note that typically that work has addressed issues, such as data cleaning, in isolation. We suggest here that the interaction between each of the steps in a template will require a systems approach to make useful progress [65]. For our next steps we intend to demonstrate the approach by developing a functional working model to be tested in a variety of real world use cases. We will then follow a series of iterative improvements towards the end goal of developing modular analysis templates into a useful tool that may be used by anyone.

\section{ACKNOWLEDGEMENTS}

- EPSRC Doctoral Training Partnership for funding this work.

- Dr. M. A. Sinclair for his many helpful suggestions during the writing of this paper.

\section{REFERENCES}

[1] D. Keim, J. Kohlhammer, G. Ellis, and F. Mansmann, Mastering The Information Age - Solving Problems with Visual Analytics. Goslar, Germany: Eurographics Association, 2010. [Online]. Available: http://diglib.eg.org/

[2] S. Sagiroglu and D. Sinanc, "Big data: A review," in 2013 International Conference on Collaboration Technologies and Systems (CTS). IEEE, may 2013, pp. 42-47. [Online]. Available: http: //ieeexplore.ieee.org/document/6567202/

[3] J. Manyika, M. Chui, B. Brown, J. Bughin, R. Dobbs, C. Roxburgh, and A. H. Byers, "Big data: The next frontier for innovation, competition, and productivity," McKinsey Global Institute, Tech. Rep. June, 2011. [Online]. Available: www.mckinsey.com/mgi.
[4] M. Castells and P. Nations, "Information Technology Globalization and Social Development f or Social Development," Information Age, vol. 9, no. 114, pp. 1477-1483, 1999. [Online]. Available: www.unrisd.orghttp://www.unrisd.org/ unrisd/website/document.nsf/462fc27bd1fce00880256b4a0060d2af/ f270e0c066f3de7780256b67005b728c/ $\backslash \backslash \$$ FILE/dp114.pdf

[5] U. Sivarajah, M. M. Kamal, Z. Irani, and V. Weerakkody, "Critical analysis of Big Data challenges and analytical methods," Journal of Business Research, vol. 70, no. August, pp. 263-286, 2017. [Online]. Available: http://dx.doi.org/10.1016/j.jbusres.2016.08.001

[6] M. Gubanov, "Polyfuse: A large-scale hybrid data fusion system," in Data Engineering (ICDE), 2017 IEEE 33rd International Conference on. IEEE, 2017, pp. 1575-1578. [Online]. Available: http://ieeexplore. ieee.org/abstract/document/7930127/

[7] P. Russom, "Big data analytics," TDWI best practices report fourth quarter, vol. 19, no. 4, pp. 1-34, 2011. [Online]. Available: https://vivomente.com/wp-content/uploads/2016/04/big-dataanalytics-white-paper.pdf

[8] K. Kambatla, G. Kollias, V. Kumar, and A. Grama, "Trends in big data analytics," Journal of Parallel and Distributed Computing, vol. 74, no. 7, pp. 2561-2573, jul 2014. [Online]. Available: https://www.sciencedirect. com/science/article/pii/S0743731514000057?via $\{\backslash \%\} 3$ Dihub

[9] L. Wang, G. Wang, and C. A. Alexander, "Big Data and Visualization: Methods, Challenges and Technology Progress," Digital Technologies, vol. 1, no. 1, pp. 33-38, 2015. [Online]. Available: http://pubs.sciepub.com/dt/1/1/7

[10] K. Mack, J. Lee, K. Chang, K. Karahalios, and A. Parameswaran, "Characterizing Scalability Issues in Spreadsheet Software using Online Forums," University of Illinois, Tech. Rep., jan 2018. [Online]. Available: http://arxiv.org/abs/1801.03829https://arxiv.org/pdf/ 1801.03829.pdf

[11] J. Giczi and K. Szőke, "Official Statistics and Big Data," Intersections, vol. 4, no. 1, p. 24, jan 2018. [Online]. Available: http://intersections. tk.mta.hu/index.php/intersections/article/view/408

[12] A. Quarteroni, "The role of statistics in the era of big data: A computational scientist' perspective," Statistics and Probability Letters, vol. 136, pp. 63-67, may 2018. [Online]. Available: https://www.sciencedirect.com/science/article/pii/S0167715218300920

[13] D. Cook and D. F. Swayne, Interactive and Dynamic Graphics for Data Analysis with R! and GGobi. Springer, 2007. [Online]. Available: http://dx.doi.org/10.1007/978-0-387-71762-3http: //link.springer.com/10.1007/978-0-387-71762-3

[14] S. Fosso Wamba, S. Akter, A. Edwards, G. Chopin, and D. Gnanzou, "How 'big data' can make big impact: Findings from a systematic review and a longitudinal case study," International Journal of Production Economics, vol. 165, pp. 234-246, jul 2015. [Online]. Available: https://www.sciencedirect.com/science/article/pii/S0925527314004253

[15] N. Elgendy and A. Elragal, "Big Data Analytics in Support of the Decision Making Process," Procedia Computer Science, vol. 100, pp. 1071-1084, jan 2016. [Online]. Available: https://www.sciencedirect.com/science/article/pii/S1877050916324206? $\left\{\backslash_{-}\right\}$rdoc $=1\{\backslash \&\}\left\{\backslash_{-}\right\}$fmt=high $\{\backslash \&\}\left\{\backslash_{-}\right\}$origin= gateway $\{\backslash \&\}\{\backslash$ \} docanchor $=\{\backslash \&\}$ md5=b8429449ccfc9c30159a5f9aeaa92ffb $\{\backslash \&$ \} dgcid=raven $\left\{\backslash_{-}\right\} \operatorname{sd}\left\{\backslash_{-}\right\}$recommender $\left\{\backslash_{-}\right\}$email

[16] D. Fisher, R. DeLine, M. Czerwinski, and S. Drucker, "Interactions with big data analytics," Interactions, vol. 19, no. 3, p. 50, may 2012. [Online]. Available: http://dl.acm.org/citation.cfm?doid=2168931. 2168943

[17] B. Shneiderman, "The eyes have it: a task by data type taxonomy for information visualizations," in Proceedings 1996 IEEE Symposium on Visual Languages, 1996, pp. 336-343. [Online]. Available: https://www.sciencedirect.com/science/article/pii/ B9781558609150500469http://ieeexplore.iee.org/document/545307/

[18] M. Bala, O. Boussaid, and Z. Alimazighi, "Extracting-TransformingLoading Modeling Approach for Big Data Analytics," International Journal of Decision Support System Technology, vol. 8, no. 4, pp. 50-69, oct 2016. [Online]. Available: http://services.igi-global.com/ resolvedoi/resolve.aspx?doi=10.4018/IJDSST.2016100104

[19] P. M. Marín-Ortega, V. Dmitriyev, M. Abilov, and J. M. Gómez, "ELTA: New Approach in Designing Business Intelligence Solutions in Era of Big Data," Procedia Technology, vol. 16, pp. 667-674, jan 2014. [Online]. Available: https://www.sciencedirect.com/science/ article/pii/S2212017314002424?via $\{\backslash \%\} 3$ Dihub 
[20] I. A. T. Hashem, I. Yaqoob, N. B. Anuar, S. Mokhtar, A. Gani, and S. Ullah Khan, "The rise of "big data" on cloud computing: Review and open research issues," Information Systems, vol. 47, pp. 98-115, jan 2015. [Online]. Available: https://www.sciencedirect.com/science/ article/pii/S0306437914001288?via $\{\backslash \%\} 3$ Dihub

[21] W. Kim, B.-J. Choi, E.-K. Hong, S.-K. Kim, and D. Lee, "A Taxonomy of Dirty Data," Data Mining and Knowledge Discovery, vol. 7, no. 1, pp. 81-99, 2003. [Online]. Available: http://link.springer.com/10.1023/A:1021564703268

[22] W. Lup Low, M. Li Lee, and T. Wang Ling, "A knowledge-based approach for duplicate elimination in data cleaning," Information Systems, vol. 26, no. 8, pp. 585-606, dec 2001. [Online]. Available: https://www.sciencedirect.com/science/article/pii/S0306437901000412

[23] J. Van Den Broeck, S. A. Cunningham, R. Eeckels, and K. Herbst, "Data cleaning: Detecting, diagnosing, and editing data abnormalities," PLOS Medicine, vol. 2, no. 10, pp. 0966-0970, oct 2005. [Online]. Available: http://www.ncbi.nlm.nih.gov/pubmed/16138788http://www. pubmedcentral.nih.gov/articlerender.fcgi?artid=PMC1198040

[24] S. Hill and R. Scott, "Developing an Approach to Harvesting, Cleaning, and Analyzing Data from Twitter Using R," Information Systems Education Journal, vol. 15, no. 3, pp. 42-54, 2017. [Online]. Available: http://isedj.org/2017-15/n3/ISEDJv15n3p42.html

[25] R. Almeida, "Semi-automatic instantiation of data cleaning operations specified at conceptual level," Ph.D. dissertation, Universidade de Trás-os-Montes e Alto Douro, 2018. [Online]. Available: http: //repositorio.utad.pt/handle/10348/8247http://hdl.handle.net/10348/8247

[26] H. Müller and J.-C. Freytag, "Problems, Methods, and Challenges in Comprehensive Data Cleansing," Professoren des Inst. Für Informatik, pp. 1-23, 2005. [Online]. Available: http://www.dbis.informatik.hu-berlin.de/fileadmin/research/papers/ techreports/2003-hub $\left\{\backslash_{-}\right\}$ib $\left\{\backslash_{-}\right\}$164-mueller.pdf

[27] L. Lin, T. Peng, and J. Kennedy, "A rule based taxonomy of dirty data," Journal on Computing, vol. 1, no. 2, pp. 140-148, 2011. [Online]. Available: http://dl6.globalstf.org/index.php/joc/article/ download/931/864http://researchrepository.napier.ac.uk/3887/

[28] J. Rammelaere and F. Geerts, "Cleaning Data with Forbidden Itemsets," in Data Engineering (ICDE), San Diego, CA, USA, 2017, p. 15. [Online]. Available: http://ieeexplore.ieee.org/abstract/document/ 7930034/

[29] S. Kandel, A. Paepcke, J. Hellerstein, and J. Heer, "Wrangler: Interactive Visual Specification of Data Transformation Scripts," in ACM Human Factors in Computing Systems (CHI), Vancouver, BC, Canada., 2011, p. 10. [Online]. Available: http://vis.stanford.edu/files/ 2011-Wrangler-CHI.pdf

[30] M. Yakout, L. Berti-Équille, and A. K. Elmagarmid, "Don't be SCAREd," in Proceedings of the 2013 international conference on Management of data - SIGMOD '13. New York, New York, USA: ACM Press, 2013, pp. 553-564. [Online]. Available: http://dl.acm.org/citation.cfm?doid=2463676.2463706

[31] D. Deng, R. Castro, F. Ziawasch, A. Sibo, A. Elmagarmid, I. F. Ilyas, S. Madden, M. Ouzzani, and N. Tang, "(jk-p)The Data Civilizer System," Cidr, 2017. [Online]. Available: http: //cidrdb.org/cidr2017/papers/p44-deng-cidr17.pdf

[32] R. Akiwatkar, "The Most Popular Languages for Data Science - DZone Big Data," p. 2, 2017. [Online]. Available: https: //dzone.com/articles/which-are-the-popular-languages-for-data-science

[33] $\mathrm{R}$ Core Team, "R: A language and environment for statistical computing. R Foundation for Statistical Computing," 2017. [Online]. Available: https://www.r-project.org/

[34] —_ "Data analysis using data.table," 2017. [Online]. Available: https:// cran.r-project.org/web/packages/data.table/vignettes/datatable-intro.html

[35] V. Stodden, F. Leisch, and R. D. Peng, Implementing reproducible research. CRC Press/Taylor and Francis, 2014.

[36] R. Peng, "The reproducibility crisis in science: A statistical counterattack," Significance, vol. 12, no. 3, pp. 30-32, jun 2015. [Online]. Available: http://doi.wiley.com/10.1111/j.1740-9713.2015.00827.x

[37] R. Gentleman and D. Temple Lang, "Statistical Analyses and Reproducible Research," Journal of Computational and Graphical Statistics, vol. 16, no. 1, pp. 1-23, mar 2007. [Online]. Available: http://www.tandfonline.com/doi/abs/10.1198/106186007X178663

[38] EPSRC, "Clarifications of EPSRC expectations on research data management ." EPSRC, Tech. Rep. October, 2014. [Online]. Available: https://epsrc.ukri.org/files/aboutus/standards/ clarificationsofexpectationsresearchdatamanagement/
[39] IEEE, “About $\begin{gathered}\text { Sharing } \\ \text { Your }\end{gathered}$ Data and $\begin{array}{r}\text { Code } \\ \text { Center," }\end{array}$ Available: http://ieeeauthorcenter.ieee.org/create-your-ieee-article/useauthoring-tools-and-ieee-article-templates/about-managing-your-data/

[40] S. Callaghan, S. Donegan, S. Pepler, M. Thorley, N. Cunningham, P. Kirsch, L. Ault, P. Bell, R. Bowie, A. Leadbetter, R. Lowry, G. Moncoiffé, K. Harrison, B. Smith-Haddon, A. Weatherby, and D. Wright, "Making Data a First Class Scientific Output: Data Citation and Publication by NERC's Environmental Data Centres," International Journal of Digital Curation, vol. 7, no. 1, pp. 107-113, mar 2012. [Online]. Available: http://www.ijdc.net/article/view/208http: //ijdc.net/index.php/ijdc/article/view/208

[41] A. Vetrò, L. Canova, M. Torchiano, C. O. Minotas, R. Iemma, and F. Morando, "Open data quality measurement framework: Definition and application to Open Government Data," Government Information Quarterly, vol. 33, no. 2, pp. 325-337, 2016.

[42] Microsoft, "Digitally sign your macro project - Office Support," 2018. [Online]. Available: https://support.office.com/en-us/article/ Digitally-sign-your-macro-project-956E9CC8-BBF6-4365-8BFA98505ECD1C01 $\{\backslash \#\}$ ID0EAABAAA=Newer $\{\backslash$ \} Versions

[43] GnuPG, "The GNU Privacy Guard," jul 2013. [Online]. Available: https://gnupg.org/

[44] The CA / Browser Forum, "Baseline Requirements for the Issuance and Management of Publicly-Trusted Certificates, v.1.0," CA / Browser Forum, Tech. Rep., 2011. [Online]. Available: https://www.cabforum. org/wp-content/uploads/Baseline $\left\{\backslash_{-}\right\}$Requirements $\left\{\backslash \backslash_{-}\right\}$V1.pdf

[45] W. Yu, M. Carrasco Kind, and R. J. Brunner, "Vizic: A Jupyterbased interactive visualization tool for astronomical catalogs," Astronomy and Computing, vol. 20, pp. 128-139, jul 2017. [Online]. Available: https://www.sciencedirect.com/science/article/pii/ S2213133716301500?via $\{\backslash \%\} 3$ Dihub

[46] B. P. Abbott, R. Abbott, T. D. Abbott, et al., "Observation of Gravitational Waves from a Binary Black Hole Merger," Physical Review Letters, vol. 116, no. 6, p. 061102, feb 2016. [Online]. Available: https://link.aps.org/doi/10.1103/PhysRevLett.116.061102

[47] LIGO Scientific Collaboration, "LIGO Open Science Center release of GW150914," 2016. [Online]. Available: https://losc.ligo.org/events/ GW150914/

[48] IEEE Authorship Series, "How to Write for Technical Periodicals and Conferences," IEEE, Tech. Rep., 2018. [Online]. Available: http://ieeeauthorcenter.ieee.org/wp-content/uploads/How-toWrite-for-Technical-Periodicals-and-Conferences.pdf

[49] J. Macfarlane, "Pandoc User's Guide," University of California, Berkeley, CA, Tech. Rep., 2017. [Online]. Available: https://pandoc. org/MANUAL.pdf

[50] Annon, "Pandoc a universal document converter https://pandoc.org," 2018. [Online]. Available: https://pandoc.org/MANUAL.html

[51] J. Wettenhall and P. Grosjean, "Message boxes in R tcltk - SciViews," 2017. [Online]. Available: http://www.sciviews.org/recipes/tcltk/TclTkmessage-boxes/

[52] J. Fox, "Rcmdr.com," 2017. [Online]. Available: http://www. rcommander.com/https://socialsciences.mcmaster.ca/jfox/Misc/Rcmdr/

[53] T. Downie, "Using the R Commander: A Point-and-Click Interface for R," Journal of Statistical Software, vol. 75, no. Book Review 3, pp. 1-4, 2016. [Online]. Available: http://www.jstatsoft.org/v75/b03/

[54] J. Fox, "Writing R Commander Plug-in Packages," R Package, Tech. Rep., 2017. [Online]. Available: https://socialsciences.mcmaster.ca/jfox/ Books/RCommander/Writing-Rcmdr-Plugins.pdf

[55] — , "Extending the R Commander by "plug in" packages," $R$ News, pp. 1-7, 2007. [Online]. Available: https: //tolstoy.newcastle.edu.au/R/e3/help/att-2806/wrapper.pdfhttp://biostat. cau.ac.kr/CRAN/doc/Rnews/Rnews $\left\{\backslash \_\right\} 2007-3 \cdot \operatorname{pdf}\{\backslash \#\}$ page $=46$

[56] _ Using the $R$ commander: A point-and-click interface for R. Chapman and Hall/CRC, sep 2016. [Online]. Available: https: //www.taylorfrancis.com/books/9781315380537

[57] C. Tenopir, S. Allard, K. Douglass, A. U. Aydinoglu, L. Wu, E. Read, M. Manoff, and M. Frame, "Data sharing by scientists: Practices and perceptions," PLOS ONE, vol. 6, no. 6, p. e21101, jun 2011. [Online]. Available: http://dx.plos.org/10.1371/journal.pone.0021101

[58] M. A. Parsons, R. Duerr, and J. B. Minster, "Data citation and peer review," pp. 297-298, 2010. [Online]. Available: http: //doi.wiley.com/10.1029/2010EO340001

[59] B. Lawrence, C. Jones, B. Matthews, S. Pepler, and S. Callaghan, "Citation and peer review of data: moving towards formal data 
publication," International Journal of Digital Curation, vol. 6, no. 2, pp. 4-37, jul 2011. [Online]. Available: http://www.ijdc.net/article/view/ 181http://www.ijdc.net/index.php/ijdc/article/view/181/265

[60] M. Szomszor and L. Moreau, "Recording and Reasoning over Data Provenance in Web and Grid Services," in On The Move to Meaningful Internet Systems 2003: CoopIS, DOA, and ODBASE, R. Meersman, Z. Tari, and D. C. Schmidt, Eds. Berlin, Heidelberg: Springer Berlin Heidelberg, 2003, pp. 603-620.

[61] Y. L. Simmhan, B. Plale, and D. Gannon, "A framework for collecting provenance in data-centric scientific workflows," in Proceedings - ICWS 2006: 2006 IEEE International Conference on Web Services. IEEE, 2006, pp. 427-434. [Online]. Available: http: //ieeexplore.ieee.org/document/4032054/

[62] D. Dongare and V. Kadroli, "Panda: Public auditing for shared data with efficient user revocation in the cloud," in Proceedings of 2016 Online International Conference on Green Engineering and Technologies. IC-GET 2016, vol. 8, no. 1, jan 2017, pp. 92-106. [Online]. Available: http://ieeexplore.ieee.org/document/6690209/

[63] M. Sintek and S. Decker, "TRIPLE - A Query, Inference, and Transformation Language for the Semantic Web," in ISWC, vol. 2342, no. 2342. Springer, Berlin, Heidelberg, 2002, pp. 364-378. [Online] Available: http://link.springer.com/10.1007/3-540-48005-6\{\_\}28http: //www.springerlink.com/index/K1C8DY8QC4BKNPX0.pdf

[64] D. W. Davies, "Applying the RSA Digital Signature to Electronic Mail," Computer, vol. 16, no. 2, pp. 55-62, feb 1983. [Online]. Available: http://ieeexplore.ieee.org/document/1654301/

[65] A. L. Ramos, J. V. Ferreira, and J. Barceló, "Revisiting the similar process to engineer the contemporary systems," Journal of Systems Science and Systems Engineering, vol. 19, no. 3, p. $321<$ last_page> 350, 2010. [Online]. Available: http://dx.doi.org/10.1007/s11518-010$5144-8$ 